\title{
Study on the Index System of Taxi Operation Monitoring Based on Multi-source Data
}

\author{
CHEN Zhi-hong \\ Beijing Municipal Transportation Operations Coordination \\ Center \\ Beijing, China \\ chenzhihong@bjjtw.gov.cn \\ SUI Li-ying, Zhong Yuan \\ Beijing Transportation Information Center \\ Beijing, China \\ suiliying@bjjtw.gov.cn, zhongyuan@bjjtw.gov.cn
}

\author{
WENG Jian-cheng \\ Beijing University of Technology \\ Beijing, China \\ youthweng@bjut.edu.cn
}

\begin{abstract}
All kinds of taxi data structure were introduced, and based on multi-source data, proposes taxi operation monitoring index system in charge of the different needs faced on traveler, industry managers and government departments. Finally, taking the multi-source data of taxi in Beijing as an example, some important indexes of the taxi operation monitoring are calculated, and the running situation of city taxi can be mastered accurately. The research results can provide important decision support for the development of taxi development strategy, improve the management of taxi industry, and promote the sustainable development of taxi.
\end{abstract}

Keywords-taxi; Operation monitoring; Index system; multisource;

\section{INTRODUCTION}

As the demand diversification of city traveler, taxi is getting more and more attention as the important part of city transportation. In recent years, the taxi industry also appeared some chaos, taxi management has become a problem that city managers must face. How to establish taxi service quality assessment system and scientific management using the advanced technology is become urgent need. Use of information technology, based on taxi related data from satellite positioning, taximeter, telephone call etc., and the paper establishes the taxi operation monitoring and analysis index system, realizes the taxi operation condition monitoring and analysis, and improves the scientific management and service level of the taxi.

\section{Multi-SOURCE Data AnAlysis}

\section{A. Satellite Positioning Data}

Satellite positioning data has the advantages that the data can achieve real time return, but a large amount of data show that GPS data accuracy is lower as compared with the taximeter. The table structure of satellite positioning data

\footnotetext{
Sponsors: Beijing Municipal Commission of Transport Professional
} Personnel Program, Beijing Nova Program (xx2013018). varies according to the different dispatching center, but its basic structure is shown in Table 1.

TABLE I. TABLE STRUCTURE OF SATELLITE POSITIONING DATA

\begin{tabular}{|c|c|c|c|}
\hline Field & Explain & Field & Explain \\
\hline ID & Identifier & LONGITUDE & Longitude \\
\hline CENTER_ID & Dispatch center & LATITUDE & Latitude \\
\hline SIM_CODE & Terminal number & SPEED & Speed \\
\hline VEHICLE_CODE & License plate number & ANGLE & Direction \\
\hline GPS_TIME & Generating time & STATE & $\begin{array}{c}\text { Passenger } \\
\text { status }\end{array}$ \\
\hline
\end{tabular}

Sample data for satellite positioning data are shown in Table 2.

TABLE II. SATELLITE POSITIONING DATA SAMPLE

\begin{tabular}{|c|c|c|c|c|c|c|c|}
\hline $\begin{array}{c}\text { UNI } \\
\text { T_N } \\
\text { AME }\end{array}$ & $\underset{\text { DE }}{\text { SIM_CO }}$ & $\begin{array}{c}\text { VEH_C } \\
\text { ODE }\end{array}$ & $\begin{array}{c}\text { GPS_TI } \\
\text { ME }\end{array}$ & $\begin{array}{c}\mathbf{L O} \\
\mathbf{N}\end{array}$ & $\begin{array}{c}\mathbf{L A} \\
\mathbf{T}\end{array}$ & $\begin{array}{c}\text { SP } \\
\text { EE } \\
\text { D }\end{array}$ & $\begin{array}{c}\text { AN } \\
\text { GL } \\
\text { E }\end{array}$ \\
\hline JKSX & $\begin{array}{c}34395098 \\
06\end{array}$ & $\begin{array}{c}\text { Jing } \\
\text { BL9833 }\end{array}$ & $\begin{array}{c}2014 / 5 / 19 \\
23: 01: 43\end{array}$ & $\begin{array}{l}116 . \\
495\end{array}$ & $\begin{array}{c}39 . \\
93 \\
4\end{array}$ & 7.2 & 11 \\
\hline JYJ & $\begin{array}{c}13321183 \\
406\end{array}$ & $\begin{array}{c}\text { Jing } \\
\text { BQ0889 }\end{array}$ & $\begin{array}{c}2014 / 5 / 19 \\
23: 57: 46\end{array}$ & $\begin{array}{l}116 . \\
456\end{array}$ & $\begin{array}{c}39 . \\
85 \\
4\end{array}$ & 76 & 134 \\
\hline $\mathrm{QH}$ & $\begin{array}{c}86136011 \\
40385\end{array}$ & $\begin{array}{c}\text { Jing } \\
\text { BL1452 }\end{array}$ & $\begin{array}{c}2014 / 5 / 20 \\
0: 21: 48\end{array}$ & $\begin{array}{l}116 . \\
521\end{array}$ & $\begin{array}{c}40 . \\
01 \\
8\end{array}$ & $\begin{array}{c}53 . \\
6\end{array}$ & 240 \\
\hline
\end{tabular}

B. taximeter data

The taximeter data includes the basic information of taxi driver, status information, etc. The taximeter data is generally divided into two kinds of real return and regular return. The basic table of the taximeter is shown in Table 3. 
TABLE III. TABLE STRUCTURE OF TAXIMETER DATA

\begin{tabular}{|c|c|c|c|}
\hline Field & Explain & Field & Explain \\
\hline ID & ID & DAY_NUM & $\begin{array}{l}\text { Days on } \\
\text { the taxi }\end{array}$ \\
\hline DEAL_TYPE & $\begin{array}{c}\text { Transaction } \\
\text { types }\end{array}$ & DAY_TIME & $\begin{array}{l}\text { Time on } \\
\text { the taxi }\end{array}$ \\
\hline TRANS_AMOUNT & $\begin{array}{c}\text { Transaction } \\
\text { amount }\end{array}$ & CAR_CODE & $\begin{array}{c}\text { License } \\
\text { plate } \\
\text { number }\end{array}$ \\
\hline DEAL_TIME & $\begin{array}{l}\text { Transaction } \\
\text { time }\end{array}$ & DRIVER_LICENSE & $\begin{array}{l}\text { License } \\
\text { number }\end{array}$ \\
\hline RECORD_NUM & Record number & PRICE & Unit Price \\
\hline AMOUNT_REC & $\begin{array}{c}\text { Receivable } \\
\text { amounts }\end{array}$ & TEAM_CODE & $\begin{array}{c}\text { Taxi team } \\
\text { number }\end{array}$ \\
\hline WAIT_TIME & Waiting time & PICKERS & $\begin{array}{l}\text { Collector } \\
\text { number }\end{array}$ \\
\hline TRAVEL_MIL & Driving Mileage & TAC_TYPE & $\begin{array}{r}\text { Taxi } \\
\text { edition }\end{array}$ \\
\hline UNTRAVEL_MIL & $\begin{array}{l}\text { No passengers } \\
\text { mileage }\end{array}$ & CREATEDATE & $\begin{array}{l}\text { Create } \\
\text { time }\end{array}$ \\
\hline DRIVERS_FLAG & Driver signs & UPDATEDATE & $\begin{array}{l}\text { Update } \\
\text { time }\end{array}$ \\
\hline
\end{tabular}

The sample data of the taximeter is shown in Table 4.

TABLE IV. TAXIMETER DATA SAMPLE

\begin{tabular}{|c|c|c|c|c|c|c|}
\hline ID & $\begin{array}{c}\text { TRANS } \\
\text { AMOU } \\
\text { NT }\end{array}$ & $\begin{array}{c}\text { DEAL_TI } \\
\text { ME }\end{array}$ & $\begin{array}{c}\text { TRAV } \\
\text { EL_M } \\
\text { IL }\end{array}$ & $\begin{array}{c}\text { UNTR } \\
\text { AVEL } \\
\text { MIL }\end{array}$ & $\begin{array}{c}\text { DAY_ } \\
\text { TIME }\end{array}$ & $\begin{array}{c}\text { CAR_ } \\
\text { CODE }\end{array}$ \\
\hline $\begin{array}{c}92486 \\
1053\end{array}$ & 2900 & $\begin{array}{c}2014 / 5 / 20 \\
11: 52: 18\end{array}$ & 115 & 7 & 113239 & $\begin{array}{c}\text { BP358 } \\
4\end{array}$ \\
\hline $\begin{array}{c}92486 \\
7437\end{array}$ & 1800 & $\begin{array}{c}2014 / 5 / 20 \\
12: 54: 54\end{array}$ & 68 & 110 & 124409 & $\begin{array}{c}\text { BQ748 } \\
0\end{array}$ \\
\hline $\begin{array}{c}92486 \\
7443\end{array}$ & 1800 & $\begin{array}{c}2014 / 5 / 20 \\
13: 08: 17\end{array}$ & 68 & 0 & 125617 & $\begin{array}{c}\text { BQ748 } \\
0\end{array}$ \\
\hline
\end{tabular}

\section{Taxi Telephone Call Data}

Taxi telephone call data is refers to the customer reserving a taxi data information by phone, web and mobile phone software etc., including using taxi time, using taxi location, license plate, destination, taxi types, the order status information. Taxi telephone call data samples are as shown in Table 5.

TABLE V. TAXI TELEPHONE CALL DATA SAMPLE

\begin{tabular}{|c|c|c|c|c|c|c|}
\hline $\begin{array}{l}\text { Using } \\
\text { taxi } \\
\text { time }\end{array}$ & $\begin{array}{c}\text { Licens } \\
\text { e plate } \\
\text { numbe } \\
\text { r }\end{array}$ & $\begin{array}{c}\text { Taxi } \\
\text { reservat } \\
\text { ion } \\
\text { channel }\end{array}$ & $\begin{array}{c}\text { Taxi } \\
\text { reserv } \\
\text { ation } \\
\text { types }\end{array}$ & $\begin{array}{c}\text { Ord } \\
\text { er } \\
\text { statu } \\
\text { s }\end{array}$ & $\begin{array}{c}\text { Using } \\
\text { taxi } \\
\text { location }\end{array}$ & $\begin{array}{c}\text { Destin } \\
\text { ation }\end{array}$ \\
\hline $\begin{array}{c}2014 / 3 / \\
31 \\
21: 15: 0 \\
5\end{array}$ & $\begin{array}{c}\text { BP942 } \\
2\end{array}$ & $\begin{array}{c}\text { telephon } \\
\mathrm{e}\end{array}$ & $\begin{array}{c}\text { Immed } \\
\text { iate }\end{array}$ & $\begin{array}{l}\text { Com } \\
\text { plete } \\
\text { order }\end{array}$ & $\begin{array}{l}\text { Cao-Qiao } \\
\text { east road }\end{array}$ & $\begin{array}{l}\text { Capital } \\
\text { Airport }\end{array}$ \\
\hline $\begin{array}{c}\text { 2014/4/ } \\
2 \\
10: 09: 0 \\
0\end{array}$ & $\begin{array}{c}\text { BM113 } \\
6\end{array}$ & $\begin{array}{c}\text { telephon } \\
\mathrm{e}\end{array}$ & $\begin{array}{c}\text { Immed } \\
\text { iate }\end{array}$ & $\begin{array}{l}\text { Com } \\
\text { plete } \\
\text { order }\end{array}$ & $\begin{array}{c}\text { Shunyi } \\
\text { Zhongjia } \\
\text { Xinyuan } \\
\text { Hotel }\end{array}$ & $\begin{array}{l}\text { Capital } \\
\text { Airport }\end{array}$ \\
\hline $\begin{array}{c}2014 / 4 / \\
2 \\
10: 16: 0 \\
0\end{array}$ & $\begin{array}{c}\text { BP067 } \\
5\end{array}$ & $\begin{array}{c}\text { telephon } \\
\text { e }\end{array}$ & $\begin{array}{c}\text { Immed } \\
\text { iate }\end{array}$ & $\begin{array}{l}\text { Com } \\
\text { plete } \\
\text { order }\end{array}$ & $\begin{array}{c}\text { Shunyi } \\
\text { Zhongjia } \\
\text { Xinyuan } \\
\text { Hotel }\end{array}$ & $\begin{array}{c}\text { West } \\
\text { Station }\end{array}$ \\
\hline
\end{tabular}

\section{Other related data}

Other related data of taxi includs the operation approval, complaints, illegal, etc.

Operating approval data includs taxi companies, company addresses, operating status, license plates, taxi models, single and double shift, etc..

Complaints data includs the source of the complaint, the complainant's name, complaint time, the taxi company, the license plate, complaint content, etc..

Illegal data includs taxi companies, inspection time, inspection sites, parties, license plates, illegal behavior, etc.

\section{E. Data preprocessing}

In order to analyze the operation monitoring of taxi by using multi-source data, the multi-source data preprocessing is needed. Firstly, all kinds of data is respectively processed, such as satellite positioning data cleaning, map matching, single passenger time is too long, single passenger kilometer is too short and so on, then will be a match between the various types of data, and finally form a better quality of taxi sample library. See Figure 1 for specific processes.

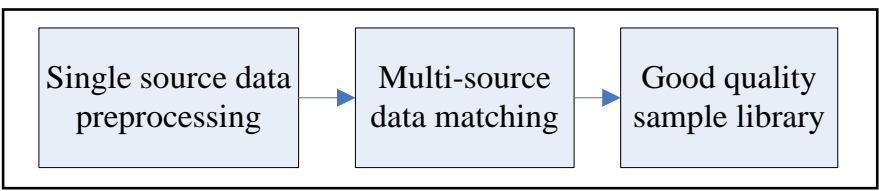

Fig. 1. Multi-source data preprocessing procedure

\section{TAXI OPERATION MONITORING INDEX SYSTEM}

Based on existing multi-source data and the key indicators, such as dispatch taxi rate, dynamic dispatch taxi rate, complaint rate, illegal rate, telephone call success rate, taxi no passengers rate, taxi spatial agglomeration, the study establishes the taxi operation monitoring index system, monitors and analyzes the taxi operation status, service quality and spatial agglomeration etc.. For different user groups, the taxi monitoring index is divided into 3 types, respectively faced on the taxi service level monitoring indicators of traveler, taxi operation level monitoring indicators of industry managers, and taxi development level monitoring indicators of government department.

\section{A. The taxi service level monitoring indicators faced on traveler}

The taxi service level monitoring indicators faced on traveler include four aspects: the convenience, the shortcut, the reliability and the safety of the taxi. Its role is to monitor and improve the taxi service level, provide high quality service for travelers. 
TABLE VI. THE TAXI SERVICE LEVEL MONITORING INDICATORS FACED ON TRAVELER

\begin{tabular}{|c|c|c|c|}
\hline Status index & Basic index & Status index & Basic index \\
\hline \multirow{4}{*}{ convenience } & $\begin{array}{c}\text { possession rate in } \\
1000 \text { peoele of taxi }\end{array}$ & \multirow{3}{*}{ reliability } & $\begin{array}{c}\text { Taxi passenger } \\
\text { speed } \\
\text { fluctuations }\end{array}$ \\
\cline { 2 - 2 } & $\begin{array}{c}\text { difficult index of } \\
\text { taking a taxi }\end{array}$ & & $\begin{array}{c}\text { Taxi time } \\
\text { fluctuations }\end{array}$ \\
\cline { 2 - 2 } & $\begin{array}{c}\text { Taxi reservation } \\
\text { success rate }\end{array}$ & \multirow{4}{*}{ safety } & $\begin{array}{c}\text { Integrity rate } \\
\text { of taxi GPS }\end{array}$ \\
\cline { 2 - 2 } shortcut & Passenger speed & & Complaint rate \\
\cline { 2 - 2 } & Average taxi time & & illegal rate \\
\hline
\end{tabular}

B. The taxi operation level monitoring indicators faced on industry managers

The taxi operation level monitoring indicators faced on industry managers mainly include dispatch taxi rate, taxi distribution density, have passenger rate, operational efficiency and operating speed etc.. Its role is to monitor and improve the taxi industry management, master the taxi operations condition provide support for formulating and adjusting the trade policy related to the taxi industry.

TABLE VII. THE TAXI OPERATION LEVEL MONITORING INDICATORS FACED ON INDUSTRY MANAGERS

\begin{tabular}{|c|c|c|c|}
\hline Status index & Basic index & $\begin{array}{l}\text { Status } \\
\text { index }\end{array}$ & Basic index \\
\hline \multirow{4}{*}{$\begin{array}{l}\text { dispatch taxi } \\
\text { rate }\end{array}$} & $\begin{array}{l}\text { dispatch taxi } \\
\text { rate in five } \\
\text { minutes }\end{array}$ & \multirow{5}{*}{$\begin{array}{c}\text { Operation } \\
\text { benefit }\end{array}$} & $\begin{array}{l}\text { Mileage } \\
\text { utilization }\end{array}$ \\
\hline & $\begin{array}{l}\text { dispatch taxi } \\
\text { rate in an hour }\end{array}$ & & Time utilization \\
\hline & $\begin{array}{l}\text { dispatch taxi } \\
\text { rate during peak } \\
\text { period }\end{array}$ & & $\begin{array}{c}\text { Average daily } \\
\text { passenger } \\
\text { mileage }\end{array}$ \\
\hline & $\begin{array}{l}\text { dispatch taxi } \\
\text { rate of a day }\end{array}$ & & $\begin{array}{l}\text { Average daily } \\
\text { assenger time }\end{array}$ \\
\hline \multirow{3}{*}{$\begin{array}{l}\text { Distribution } \\
\text { density }\end{array}$} & $\begin{array}{c}\text { Distribution } \\
\text { density in five } \\
\text { minutes }\end{array}$ & & $\begin{array}{l}\text { Taxi demand } \\
\text { intensity }\end{array}$ \\
\hline & $\begin{array}{c}\text { Distribution } \\
\text { density in an } \\
\text { hour }\end{array}$ & \multirow{2}{*}{$\begin{array}{l}\text { Operating } \\
\text { speed }\end{array}$} & Passenger speed \\
\hline & $\begin{array}{c}\text { Distribution } \\
\text { density of no } \\
\text { passenger taxi }\end{array}$ & & $\begin{array}{l}\text { no passengers } \\
\text { driving speed }\end{array}$ \\
\hline \multirow{3}{*}{$\begin{array}{l}\text { Have } \\
\text { passenger } \\
\text { rate }\end{array}$} & $\begin{array}{l}\text { Have passenger } \\
\text { rate in five } \\
\text { minutes }\end{array}$ & \multirow{3}{*}{\multicolumn{2}{|c|}{$\begin{array}{l}\text { Number of no passenger taxi } \\
\text { in key station }\end{array}$}} \\
\hline & $\begin{array}{l}\text { Have passenger } \\
\text { rate in an hour }\end{array}$ & & \\
\hline & $\begin{array}{c}\text { Taxi time } \\
\text { utilization }\end{array}$ & & \\
\hline
\end{tabular}

C. The taxi development level monitoring indicators faced on government department

The taxi development level monitoring indicators faced on government department mainly include four aspects, such as development scale, overall situation, travel characteristics and profit characteristics and so on. Its role is to help the government departments monitor and manage taxis better, provide important support for government decision-making.
TABLE VIII. THE TAXI DEVELOPMENT LEVEL MONITORING INDICATORS FACED ON GOVERNMENT DEPARTMENT

\begin{tabular}{|c|c|c|c|}
\hline Status index & Basic index & Status index & Basic index \\
\hline \multirow{4}{*}{$\begin{array}{l}\text { Development } \\
\text { scale }\end{array}$} & $\begin{array}{l}\text { Total number } \\
\text { of taxis in the } \\
\text { city }\end{array}$ & \multirow{3}{*}{$\begin{array}{c}\text { Travel } \\
\text { characteristic }\end{array}$} & $\begin{array}{c}\text { Average time } \\
\text { consuming of } \\
\text { single passenger }\end{array}$ \\
\hline & $\begin{array}{c}\text { possession } \\
\text { rate in } 1000 \\
\text { people of taxi }\end{array}$ & & $\begin{array}{c}\text { Average distance } \\
\text { of single } \\
\text { passenger }\end{array}$ \\
\hline & $\begin{array}{c}\text { Taxi platform } \\
\text { density }\end{array}$ & & $\begin{array}{c}\text { Daily passenger } \\
\text { number }\end{array}$ \\
\hline & $\begin{array}{l}\text { Taxi daily } \\
\text { emissions }\end{array}$ & \multirow{4}{*}{$\begin{array}{c}\text { Profit } \\
\text { characteristic }\end{array}$} & Business income \\
\hline \multirow{3}{*}{$\begin{array}{l}\text { Overall } \\
\text { situation }\end{array}$} & $\begin{array}{l}\text { Average taxi } \\
\text { dispatch rate }\end{array}$ & & $\begin{array}{c}\text { Average amount } \\
\text { of single } \\
\text { passenger }\end{array}$ \\
\hline & $\begin{array}{c}\text { Daily } \\
\text { operating } \\
\text { mileage } \\
\end{array}$ & & Operating costs \\
\hline & $\begin{array}{c}\text { Daily } \\
\text { operating time }\end{array}$ & & \\
\hline
\end{tabular}

\section{APPLICATION EXAMPLES}

Taking taxi in Beijing city zone as the analysis object, a part of above indexes are calculated and analyzed. To analyze the daily data on March 25, 2015, the number of taxi operations was 63.2 thousand; daily dispatch taxi rate during peak period was $87.02 \%$, the dispatch taxi rate of early peak was $60.75 \%$, the dispatch taxi rate of late peak was $68.82 \%$; diurnal hours operation taxi number peak was 52.9 thousand, appeared at 11:00-12:00; taxi telephone call success orders was 238.3 thousand, the success rate was $67.99 \%$.

March 16-22, 2015, the daily average operating taxi number was 62.2 thousand, among them, the daily average operating taxi numb within five ringer was 58.3 thousand; the weekday daily peak dispatch taxi rate was $78.92 \%$, early peak dispatch taxi rate daily was $60.00 \%$, late peak dispatch taxi rate daily was $61.95 \%$; in weekend, daily average late peak dispatch taxi rate was $86.72 \%$, early peak dispatch taxi rate daily was $59.52 \%$, late peak dispatch taxi rate daily was $69.17 \%$.

March 2015, the single taxi passenger mileage of 156 kilometers, the average taxi no passenger mileage was 84 kilometers; double shift taxi passenger taxi mileage was 208 kilometers, the average taxi no passenger mileage was 106 kilometers. The number of single shift average taxi having passenger was 17 times; The number of double shift average taxi having passenger was 23 times.

\section{CONCLUSION}

The multi-source, such as satellite positioning, taximeter, telephone call etc., has more abundant and more accurate information than the traditional single data source. Through preprocessing and analyzing the multi-source data, the taxi operation monitoring index can be accurately retrieved, including dispatch taxi number, dynamic dispatch rate, telephone call success rate, no passenger taxi rate, operational mileage and so on. Based on the available static and dynamic indexes, the taxi operation monitoring index system is built faced on traveler, industry manager and government department, which analyzes operation, service and 
development level of taxi. On the basis of it, taking the data of Beijing City as an example, some important indicators are calculated, which provide an important decision support for accurately grasping the operation of urban taxi, drafting taxi development strategy, improving the taxi industry management, promoting the sustainable development of taxi.

\section{References}

[1] HU Xiao-wen, Feng Jun-jia. Research on characteristics of taxi traffic based on GPS data[J]. Urban Transport of China, 2007,5(2) , pp. 91-95.

[2] WENG Jian-cheng, RONG Jian, YU Quan, REN Fu-tian. Optimization on estimation of travel speed based on the real-time floating car data[J]. Journal of Beijing University of Technology, 2007,33(5) , pp. 459-464.
[3] ZHANG Xiao-liang, CHEN Zhi-hong, LIU Dong-mei, et al. A Taxi Travel Forecasting Method Based on Multi-source Data[J]. Journal of Road Traffic \& Safety, 2015, 15(1) , pp. 47-51

[4] WENG Jian-cheng, Liu Wen-tao, CHEN Zhihong, RONG Jian. Research on Floating Car Data Based Taxi Operation and Management[J]. Journal of Beijing University of Technology, 2010,36(6) , pp. 779-784.

[5] CHI Guang-hua. Analysis of taxi operational features based on floating car data [J]. Journal of Transpo Worldc, 2011(20) , pp. 84-85.

[6] BAI Zhu, WANG Jian, HU Xiao-wei. Operational Efficiency Evaluation of Urban Taxi System[J]. Journal of Transportation Systems Engineering and Information Technology, 2014, 14(3) , pp. 227-233.

[7] BAI Zhu, JIN Xiao-hong. Research on the application of taxi GPS data[J]. Journal of Heilongjiang Institute of Technology, 2014(2) , pp. 50-54. 